\title{
ECONOMIC AND ENERGETIC EFFICIENCY OF COMPLEX BACTERIZATION IN SOYBEAN CULTIVATION
}

\section{Y. M. Khalep, S. F. Kozar}

Inoculants based on beneficial soil microorganisms are able to ensure the formation of effective functional plant-microbial system with high performance. Our previous studies showed that the complex pre-treatment of soybean seeds with rhizobia Bradyrhizobium japonicum and associative diazotrophs Azospirillum brasilense contributes to the increase in the number and weight of nodules and increase in activity of nitrogen fixation in the root zone of plants. It was proved that the use of mixed cultures of nitrogen fixing microorganisms can increase soybean yield in comparison with inoculation using pure culture of rhizobia.

At the same time, one of the main criteria for assessment of any industrial product or agricultural measure is the economic efficiency of its use. This particular guided the majority of agricultural producers in choosing a certain product in a great variety of innovations on the market

Important vector for the assessment of developed innovation, predominantly in the national economic aspect, also is an analysis of energetic efficiency, especially given the fact that economic parameters cannot always be objective as they are influenced by a number of external factors (inflation, market conditions, etc.).

For this purpose, we studied the influence of various inoculants based on B. japonicum and A. brasilense in soybean cultivation technology on the key parameters of economic and energetic efficiency of

Materials and methods. The basis of calculation included average parameters of yield obtained in the field experiments in 2011-2013. The efficiency of inoculants based on B. japonicum M-8, A. brasilense 18-2, A. brasilense 410 cultivated in pure and mixed cultures was tested for soybean varieties Lehenda during cultivation on the meadow black soil. Planning and conducting of field experiments, accounting of crop and statistical processing of findings was performed using conventional methods.

Scheme of field experiments:

1. Control (without bacterization).

2. Preplant bacterization of soybean seeds with B. japonicum M-8.

3. Preplant bacterization with A. brasilense 410 .

4. Preplant bacterization with $A$. brasilense 18-2.

5. Preplant bacterization with B. japonicum M-8 and A. brasilense 410.

6. Preplant bacterization with B. japonicum M-8 and A. brasilense 18-2. 
Measurement of the parameters of economic efficiency was performed using methods and conventional methodological approaches based on comparing the results of certain agricultural measure with costs of its application. In this regards, we have analyzed such basic parameters of economic efficiency: unit cost, profit, level of profitability, return on additional costs. During establishment of the costs associated with bacterization, we considered changing not only those parameters that are directly related to inoculation (direct costs: cost of inoculant, cost of bacterization, cost of revision and transport of extra yield, etc.), as well as changes in overhead costs, which in costing of products are distributed in proportion to direct. For this purpose, we calculated the full cost of product, since profit, as one of the final parameters of economic efficiency, is the difference between the price and the total cost of product. Use of such methodological and methodical approach slightly increases the estimated level of spending on bacterization, but at the same time, promotes objective evaluation of the economic efficiency of this agricultural measure.

Since field studies were conducted on small plots, we applied modelling process for technological losses in terms of production scale using standard technologies to determine the cost-effectiveness of different variants of the experiment. Technological operations, resource consumption and calculation algorithm of product was accepted according to the method of NSC "Institute of Agrarian Economy" of the NAAS with the inclusion of additional costs associated with the use of studied inoculants. Prices for resources and agricultural products were set up at the average level in 2015.

To determine the energetic efficiency of bacterization, costs of all types of material resources and obtained yield were converted to energetic equivalents according to the methods.

Summarizing conducted analysis of the impact of pre-plant bacterization on economic and energetic efficiency of soybean production, it should be noted that various variants of inoculants based on rhizobia $B$. japonicum and associative diazotrophs $A$. brasilense show differing results. Thus, variants with complex bacterization are characterized by higher levels of both economic and energetic efficiency compared to the variant without inoculation and variant with a pure culture of rhizobia, thus, the highest level of efficiency is provided by the combined use of B. japonicum M-8 and A. brasilense 410. The above is the result of delaying growth of expenditure associated with the use of inoculants based on these microorganisms, compared with an increase of grain yield. At the same time, no positive impact of A. brasilense, applied separately, was found in terms of the studied parameters, or they were at a minimum level. 